Oper Orthop Traumatol 2020 32:1 https://doi.org/10.1007/s00064-019-00644-9 Online publiziert: 6. Dezember 2019 (c) Springer Medizin Verlag $\mathrm{GmbH}$, ein Teil von Springer Nature 2019

\section{Dank an die Gutachterinnen und Gutachter 2019}

Für die Qualität und Objektivität der Beiträge sind neben den engagierten Autorinnen und Autoren auch die vielen qualifizierten Gutachterinnen und Gutachter maßgeblich, die im Rahmen des Peer-Review-Prozesses die Manuskripte inhaltlich-wissenschaftlich prüfen und Empfehlungen zur konkreten Verbesserung äußern. Schriftleitung und Redaktion von Operative Orthopädie und Traumatologie danken allen Gutachterinnen und Gutachtern, die im vergangenen Jahr Manuskripte für diese Zeitschrift begutachtet haben, sowie allen Schwerpunktherausgebern herzlich für die konstruktive und gewissenhafte Arbeit. Durch ihren Einsatz garantieren sie die hohe Qualität und klinische Relevanz der OOT.
Matthias Knobe, Aachen

Philipp Kobbe, Aachen

Hendrik Kohlhof, Bonn

Andreas Korge, München

Martin Langer, Münster

Jon Largiader, Zürich

Djordje Lazovic, Oldenburg

Luke Leenen, Utrecht

Wolfgang Lehmann, Göttingen

Wolfgang Linhart, Heilbronn

Steffen Löw, Bad Mergentheim

Uwe Maus, Oldenburg

Michael Mayer, München

Peter Messmer, Zürich

Wolfgang Miehlke, Pforzheim

Gunnar Müller, Hamburg

Ulrich Nöth, Berlin

Sabine Ochman, Münster

Luca Papavero, Hamburg

Carsten Perka, Berlin

Thomas Pillukat, Bad Neustadt an der

Saale

Christian Plaaß, Hannover

Stefan Rammelt, Dresden

Peter Richter, Ulm

Bernd Roetman, Rheine

Maximilian Rudert, Würzburg

Oliver Rühmann, Laatzen

Philipp Schleicher, Frankfurt

Andreas Schmitt, München

Saskia Schnabl, Tübingen
Klaus Schnake, Fürth

Steffen Schroeter, Tübingen

Franz Josef, Seibert Graz

Christoph Siepe, München

Christian Spies, Bad Rappenau

Andre Steinert, Hausham

Hartmut Stinus, Northeim

Markus Tingart, Aachen

Lennart Viezens, Göttingen

Stephan Vogt, Augsburg

Urs von Deimling, Sankt Augustin

Rüdiger von Eisenhart-Rothe,

München

Jan von Recum, Ludwigshafen

Matthias Wimmer, Bonn

Johannes Zeichen, Minden
Florian Imhoff, München

Bernd Kinner, Stuttgart

Stephan Kirschner, Karlsruhe

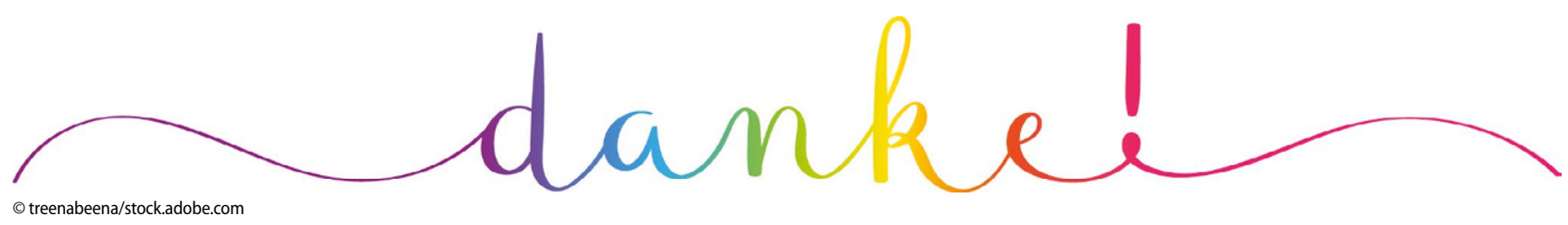

